\title{
Cell cycle-dependent localization and possible roles of the small GTPase Ran in mouse oocyte maturation, fertilization and early cleavage
}

\author{
Yun-Kao Cao ${ }^{1,2}$, Zhi-Sheng Zhong ${ }^{1}$, Da-Yuan Chen ${ }^{1}$, Gui-Xue Zhang ${ }^{2}$, Heide Schatten ${ }^{3}$ and \\ Qing-Yuan Sun ${ }^{1}$ \\ ${ }^{1}$ State Key Laboratory of Reproductive Biology, Institute of Zoology, The Chinese Academy of Sciences, No. 25 \\ Beisihuanxi Road, Beijing 100080, China, ${ }^{2}$ Department of Animal Science and Technology, Northeast Agricultural \\ University, Harbin, China and ${ }^{3}$ Department of Veterinary Pathobiology, University of Missouri, Columbia, \\ Missouri, USA
}

Correspondence should be addressed to Q-Y Sun; Email: sunqy@ioz.ac.cn; sunqy1@yahoo.com

\begin{abstract}
The small GTPase Ran controls numerous cellular processes of the mitotic cell cycle. In this experiment, we investigated the localization and possible roles of Ran during mouse oocyte meiotic maturation, fertilization and early cleavage by using confocal laser scanning microscopy, antibody microinjection and microtubule disturbance. The results showed that Ran was localized mainly in the nucleus (except for the nucleolus) in the oocyte, zygote and early embryo. At pro-metaphase of meiosis I, Ran distributed throughout the cell, but predominantly concentrated around the condensed chromosomes. During the completion of meiosis I and meiosis II, it concentrated to the meiotic spindle microtubules except for the midbody region. After sperm penetration, Ran dispersed with the extrusion of the second polar body and gradually concentrated in the male and female pronuclei thereafter. Ran was also observed to exist diffusely in the cytoplasm in prophase; it concentrated at the mitotic spindle, and migrated to the nucleus during early cleavage. Ran's concentration around the spindle disappeared when microtubule assembly was inhibited by colchicine, while it was concentrated around the chromosomes after microtubule stabilization with taxol treatment. Ran did not display any role in cytokinesis during division when pseudo-cleavage of germinal vesicle-intact oocytes was induced. Anti-Ran antibody microinjection decreased the germinal vesicle breakdown and the first polar body extrusion, and distorted spindle organization and chromosome alignment. Our results indicate that Ran has a cell cycle-dependent localization and may have regulatory roles in cell cycle progression and microtubule organization in mouse oocytes, fertilized eggs and early embryos.

Reproduction (2005) $130431-440$
\end{abstract}

\section{Introduction}

The $24 \mathrm{kDa}$ small GTPase Ran (Ras-related nuclear) (Bischoff \& Ponstingl 1991a, 1992b, Takai et al. 2001), whose cDNA sequence codes for 216 amino acid residues, was originally identified as TC4 by screening a human teratocarcinoma cDNA library with degenerate oligonucleotides based on the conserved DTAGQE sequence of Ras family members (Drivas et al. 1990, Matsumoto \& Beach 1991). Unlike other Ras-related proteins, Ran does not contain a cysteine residue close to the C-terminus, known as the acceptor site for post-translational prenylation in most other Ras-related proteins. Instead, it has an acidic C-terminal sequence -DEDDDL - which is essential for Ran's function (Lounsbury et al. 1994, Ren et al.
1994). Ran was found to be the most abundant Rasrelated protein in HeLa cells, where it represents $0.4 \%$ of total cell protein.

The Ran GTPase affects many cellular processes, including the regulation of cell cycle progression, nuclear envelope structure and function, and nucleo-cytoplasmic transport. Ran is regulated by a cytosolic-activating protein, RanGAP1, and by a chromatin-bound nucleotide exchange factor, RCC1. The distribution of RanGTP provides important spatial information that directs cellular activities during different parts of the cell cycle (Sazer \& Dasso 2000, Dasso 2001).

During interphase, the localization of RCC1 and RanGAP1 implies that nuclear Ran is GTP-bound and cytosolic Ran is GDP-bound. This compartmentalization 
determines the direction of nuclear transport by promoting the loading and unloading of transport receptors in a manner that is appropriate to the nucleus or cytosol, that is, the nuclear RanGTP promotes the dissociation of import complexes - and hence the release of nuclear proteins in the nucleoplasm - as well as the assembly of export complexes, which, conversely, mediate transport of cytoplasmic proteins and RNAs to the cytoplasm (Gorlich \& Kutay 1999).

In mitosis the nuclear envelope breaks down and condensed chromosomes are released into the cytoplasm, and then spindle microtubules are nucleated by centrosomes and chromosomes (Compton 2000, Wittmann et al. 2001). Strikingly, addition of RanGTP or mutant forms of Ran locked in a GTP-like conformation to meiotic Xenopus egg extracts stimulates microtubule assembly and can even induce the formation of centrosome-independent bipolar spindles in the presence or absence of chromatin (Carazo-Salas et al. 1999, Kalab et al. 1999, Ohba et al. 1999, Zhang et al. 1999). The role of Ran in spindle formation has also been demonstrated in yeast and Caenorhabditis elegans (Ouspenski 1998, Fleig et al. 2000, Quimby et al. 2000, Bamba et al. 2002).

When adding chromatin to Xenopus egg extract in vitro, it was found that either RanGTP or RanGDP together with RCC1 is necessary for early membrane fusion steps in nuclear envelope assembly (Hetzer et al. 2000). Similarly, beads coated with wild-type Ran formed a pseudonucleus surrounded by nuclear envelope, whereas beads attached to mutant forms of Ran incapable of cycling between GTP- and GDP-bound conformations failed to do so (Zhang \& Clark 2000). More recently, it was reported that perturbing the Ran system in C. elegans by double-stranded RNA-mediated interference (RNAi) gave rise to embryos where the chromatin no longer associated with nucleoporins, indicative of either a defect in nuclear envelope assembly or nuclear pore complex (NPC) assembly or insertion into the nuclear envelope (Bamba et al. 2002).

Mammalian oocytes are arrested at the $\mathrm{G}_{2} / \mathrm{M}$ phase transition of the first meiotic division. In vitro, fully grown oocytes liberated from their follicles spontaneously reinitiate meiosis I, characterized by germinal vesicle (GV) breakdown (GVBD), chromatin condensation, spindle assembly, emission of the first polar body and progression to metaphase of the second meiotic division (MII), at which stage they undergo a second arrest until fertilization. After sperm penetrates the oocyte, the second polar body extrudes, male and female pronuclei form and syngamy occurs to start early embryo development. Ran is highly conserved in eukaryotes from yeast to humans, but we still do not know whether such an important GTPase regulates cellular processes from a follicular oocyte to an early embryo. In the present study, we investigated: (i) the distribution of Ran during meiotic maturation, fertilization and subsequent early embryo development; (ii) the correlation of microtubule assembly and Ran localization in mouse oocytes after different treatments with microtubule regulators or after anti-Ran antibody microinjection; and (iii) Ran's distribution in pseudo-cleavage of GV-intact oocytes. All these experiments were aimed at clarifying the possible correlation between Ran and microtubule assembly as well as cell cycle progression in mouse oocytes.

\section{Materials and Methods}

\section{Chemicals}

All chemicals used in this experiment were purchased from Sigma Chemical Company (St Louis, MO, USA) except for those specifically mentioned. Drugs were prepared as stock solutions by dissolving in dimethyl sulfoxide and stored in a dark box at $-20^{\circ} \mathrm{C}$. The stock solutions were diluted with M2 medium (Sigma) prior to use.

\section{Anti-Ran antibody}

Anti-Ran antibody (c-20) was purchased from Santa Cruz Biotechnology, Inc. (Santa Cruz, CA, USA). It was an affinity-purified goat polyclonal antibody raised against a peptide mapping to the C-terminus of Ran of human origin, and it reacts with Ran of mouse, rat and human origin.

\section{Oocyte collection and culture}

Kunming strain mice at 4-6 weeks of age were purchased from the Laboratory Animal Core, Institute of Genetics, Chinese Academy of Sciences. Animals were housed in a temperature-controlled room with proper darkness-light cycles, fed with a regular diet, and maintained under the care of the Laboratory Animal Unit, State Key Laboratory of Reproductive Biology, Institute of Zoology, the Chinese Academy of Sciences. Following 5 days of acclimation, $\mathrm{GV}$-stage oocytes were collected from ovaries of the mice $48 \mathrm{~h}$ after the females were injected with $10 \mathrm{IU}$ equine gonadotropin (eCG). GV-intact follicular oocytes were released from the large antral follicles by puncturing with a needle in M2 medium with $60 \mu \mathrm{g} / \mathrm{ml}$ penicillin and $50 \mu \mathrm{g} / \mathrm{ml}$ streptomycin. Oocytes were denuded by pipetting and normal-looking oocytes were cultured for maturation in $\mathrm{M} 2$ at $37^{\circ} \mathrm{C}$ in a humidified atmosphere of $5 \% \mathrm{CO}_{2}$.

Cumulus cell-enclosed MIl-arrested eggs were obtained from mice of the same strain. Females were superovulated by i.p. injection with $10 \mathrm{IU}$ eCG, and $48 \mathrm{~h}$ later they were injected with $10 \mathrm{IU}$ human gonadotropin (hCG). Mice were killed by cervical dislocation $15 \mathrm{~h}$ after hCG injection. The cumulus cell masses surrounding the eggs were removed by brief exposure to $300 \mathrm{IU} / \mathrm{ml}$ hyaluronidase in M2 medium. Oocytes were subjected to in vitro fertilization and parthenogenetic activation by $7 \%$ alcohol for $5 \mathrm{~min}$, and then cultured in M2 medium for $6 \mathrm{~h}$. 


\section{Zygote and embryo collection}

In vivo fertilized zygotes were collected $16 \mathrm{~h}$ after hCG from the oviduct ampullae of superovulated females that had been mated with the same strain of males. After removing cumulus cells with $300 \mathrm{IU} / \mathrm{ml}$ hyaluronidase in M2 medium, zygotes were cultured in M16 medium (Sigma) until use. Two cell embryos were flushed from the oviducts of copulated mice $44-46 \mathrm{~h}$ after hCG injection and cultured in M2 medium. Embryos at different stages of mitosis were collected for confocal microscopy.

\section{Confocal microscopy}

Ran detection was based on the procedures reported previously (Melchior et al. 1995). After removal of zona pellucidae (ZP) in acidified Tyrode's solution ( $\mathrm{pH}$ 2.5) eggs were fixed in $4 \%$ formaldehyde in PBS for $30 \mathrm{~min}$, and then incubated in incubation buffer $(0.5 \%$ Triton X-100 in $20 \mathrm{mM}$ HEPES, pH 7.4, $3 \mathrm{mMMgCl}_{2}, 50 \mathrm{mM} \mathrm{NaCl}$, $300 \mathrm{mM}$ sucrose, $0.02 \% \mathrm{NaN}_{3}$ ) for $30 \mathrm{~min}$, washed in PBS with $0.1 \%$ Tween 20 and $0.01 \%$ Triton X-100, then incubated with 1:100 of the polyclonal anti-Ran antibody at $4{ }^{\circ} \mathrm{C}$ overnight. The eggs were rinsed three times, each for $5 \mathrm{~min}$, and incubated with 1:100 FITC-conjugated rabbit anti-goat IgG for $1 \mathrm{~h}$, followed by staining with $10 \mu \mathrm{g} / \mathrm{ml}$ propidium iodide. Finally the eggs were mounted on glass slides and examined using a TCS-4D laser scanning confocal microscope (Leica Microsystems, Bensheim, Germany).

Tubulin detection was conducted by fixing and permeabilizing the eggs in methanol for $5 \mathrm{~min}$ at $-20^{\circ} \mathrm{C}$, and incubating the eggs in 1:50 diluted FITC-anti- $\alpha$-tubulin for $1 \mathrm{~h}$, then staining with propidium iodide as described above.

\section{Microinjection of anti-Ran antibody}

Anti-Ran antibody $(200 \mu \mathrm{g} / \mathrm{ml}$ in $1 \mathrm{ml}$ PBS containing $0.1 \% \mathrm{NaN}_{3}$ and $0.2 \%$ gelatin) was injected into the cytoplasm of fully grown GV oocytes as described by Dai et al. (2000). The injection was repeated three times, and 35 oocytes were used each time. An Eppendorf microinjector (Hamburg, Germany) was used for these experiments. All microinjections were performed by using a beveled micropipette to minimize damage and were finished within $30 \mathrm{~min}$. A microinjection volume of about $7 \mathrm{pl}$ per oocyte was used in all the experiments. The same amount of goat IgG diluted in PBS containing $0.1 \% \mathrm{NaN}_{3}$ and $0.2 \%$ gelatin was injected as control. After injection, eggs were washed thoroughly with M2 medium and cultured in the same medium.

\section{Experimental design}

\section{Experiment 1}

To investigate the expression of Ran during mouse oocyte meiotic maturation, fertilization, and early embryo mitosis, oocytes (eggs) at different stages of meiosis, fertilization or early development were collected for Ran localization with confocal microscopy.

\section{Experiment 2}

To further reveal the relationship between the spindle assembly/disassembly and the location of Ran, MII oocytes were treated with $1 \mu \mathrm{M}$ microtubule disassembly inhibitor taxol for $10 \mathrm{~min}$ or $10 \mu \mathrm{g} / \mathrm{ml}$ microtubule polymerization inhibitor colchicine for $1 \mathrm{~h}$ at $37^{\circ} \mathrm{C}$. After each treatment, oocytes were collected for confocal microscopy.

\section{Experiment 3}

To investigate the role of Ran in cytoplasmic cleavage (cytokinesis), induction of pseudo-cleavage was performed as reported (Wassarman et al. 1977). Briefly, cumulus-free and GV-intact oocytes were cultured in M2 medium containing $0.2 \mathrm{mM}$ 3-isobutyl-1-methyl-xanthine (IBMX) (this drug is used to prevent spontaneous meiotic resumption) and $5 \mu \mathrm{M}$ cytochalasin $\mathrm{B}(\mathrm{CB})$ overnight. To increase the rate of pseudo-cleaved oocytes, some ZP-free oocytes were cultured under the same conditions.

\section{Experiment 4}

The possible roles of Ran in meiosis spindle organization were revealed by antibody microinjection. Anti-Ran antibody was injected into GV-intact oocytes. The GVBD rate was recorded $2 \mathrm{~h}$ after culture. The spindle formation and polar body extrusion rate was recorded after $14 \mathrm{~h}$ of injection. The spindle structure of the oocytes undergoing GVBD was examined at $8 \mathrm{~h}$ and extrusion of the first polar body was examined at $14 \mathrm{~h}$ after injection by confocal microscopy.

\section{Statistical analysis}

All data on the GVBD rate of oocytes after antibody microinjection were evaluated by $\chi^{2}$ analysis. Eggs showing degenerative signs were not included.

\section{Results}

\section{Subcellular localization of Ran in mouse oocytes, fertilized eggs and early embryos}

The localization of Ran varied at different developmental stages during oocyte maturation and fertilization as well as mitotic division. In GV oocytes, Ran was localized mainly in the entire GV except for the nucleolus, and no obvious green fluorescence was observed in the cytoplasm (Fig. 1A). After GVBD, Ran dispersed throughout the entire cell, but it was not evenly distributed. There were numerous concentrated dots around the condensed chromatin or chromosomes (Fig. 1B-D). With the organization of chromosomes to the equatorial plate, Ran staining was preferentially distributed to the spindle. It was associated 
with the spindle microtubules, while it distributed evenly to other areas of the oocyte (Fig. 1E and F). At anaphase I and telophase I following the separation of chromosomes, Ran accumulated in the separating region between the first polar body and the oocyte, but staining was not observed in the midbody (Fig. 1G). Immediately after meiosis I, Ran concentrated to the MII spindle microtubules (Fig. 1H).

After sperm penetration, the oocyte was released from the MII arrest and completed the meiosis. Ran was found to diffuse within the cytoplasm, but concentrated in the second polar body (Fig. 2A). With the expansion of sperm chromatin, Ran began to move to the region around the expanding sperm chromatin and oocyte chromatin, and concentrated to early male and female pronuclei (Fig. 2B). With the growth of pronuclei the green fluorescent staining of Ran became increasingly stronger in the pronuclei as compared with the cytoplasm (Fig. 2C). When the male and female pronuclei became apposed, a significant signal of Ran was observed in the pronuclei (Fig. 2D and E). After pronuclear membrane breakdown, Ran dispersed through the entire zygote cytoplasm, but again it was not evenly distributed. Numerous concentrated dots were observed in the chromosome region (Fig. 2F).

When the two masses of chromosomes converged together Ran was preferentially stained in this region (Fig. 3A and B). When chromosomes became organized to the equatorial plate, Ran was stained in association
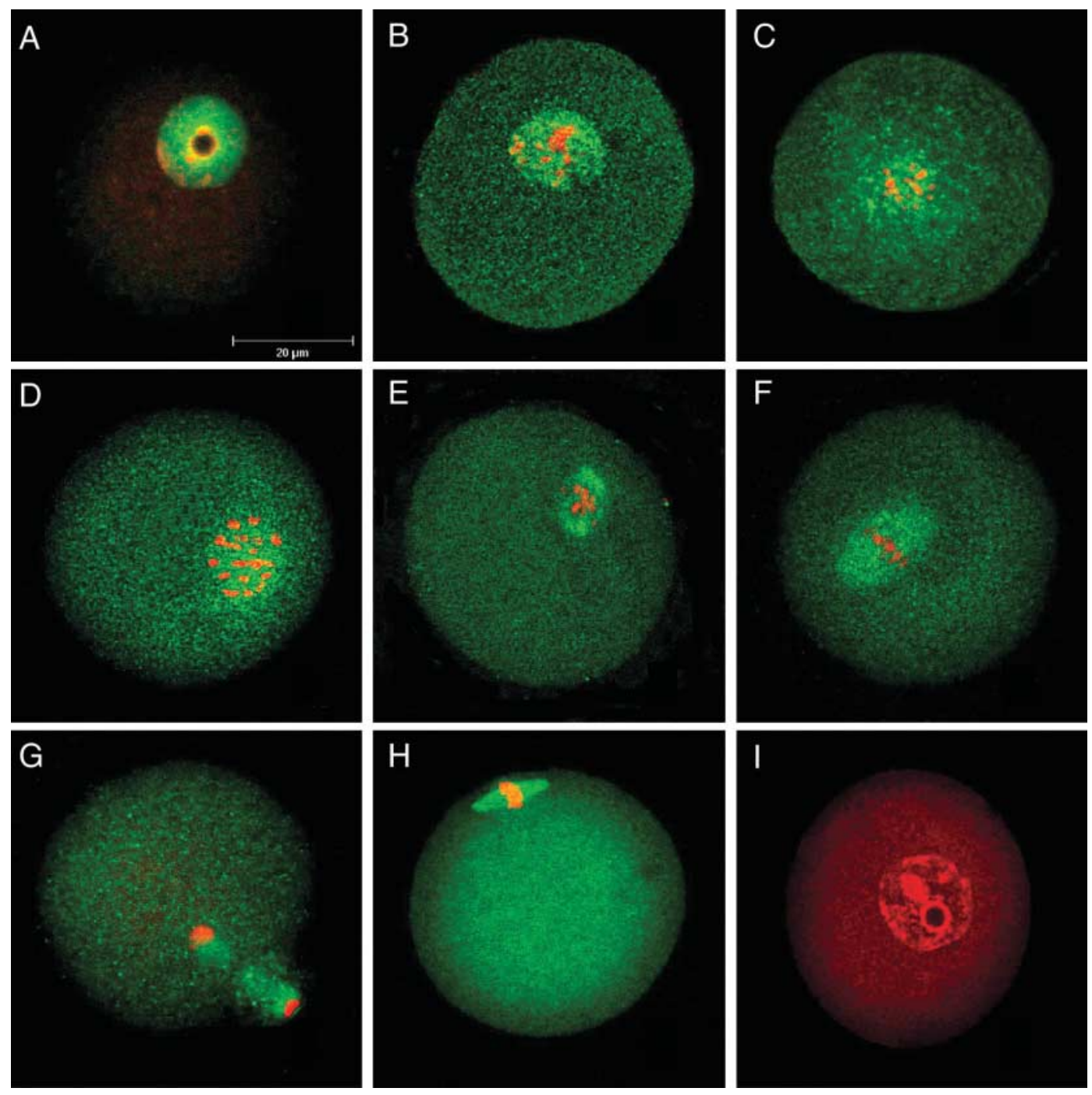

Figure 1 Immunofluorescent localization of Ran during meiotic maturation. Green, Ran; red, chromatin; yellow, overlapping of green and red. Ran localized mainly to the entire GV except the nucleolus. No obvious green fluorescence in the cytoplasm was observed (A). When GVBD occurred, Ran dispersed throughout the entire oocyte, the area around the condensed chromatin was preferentially stained with Ran (B-D). At metaphase I, with the organization of chromosomes to the equatorial plate, Ran was associated with the spindle microtubules (E and F). At telophase I, Ran accumulated to the separated spindle between the first polar body and the oocyte except for the midbody (G). At MII Ran was concentrated on the MII spindle (H). A GV oocyte was used as a negative control for Ran confocal microscopy, in which no first antibody was used but the fluorescent second antibody was used just as in the experimental group (I). Original magnification $\times 630$. 

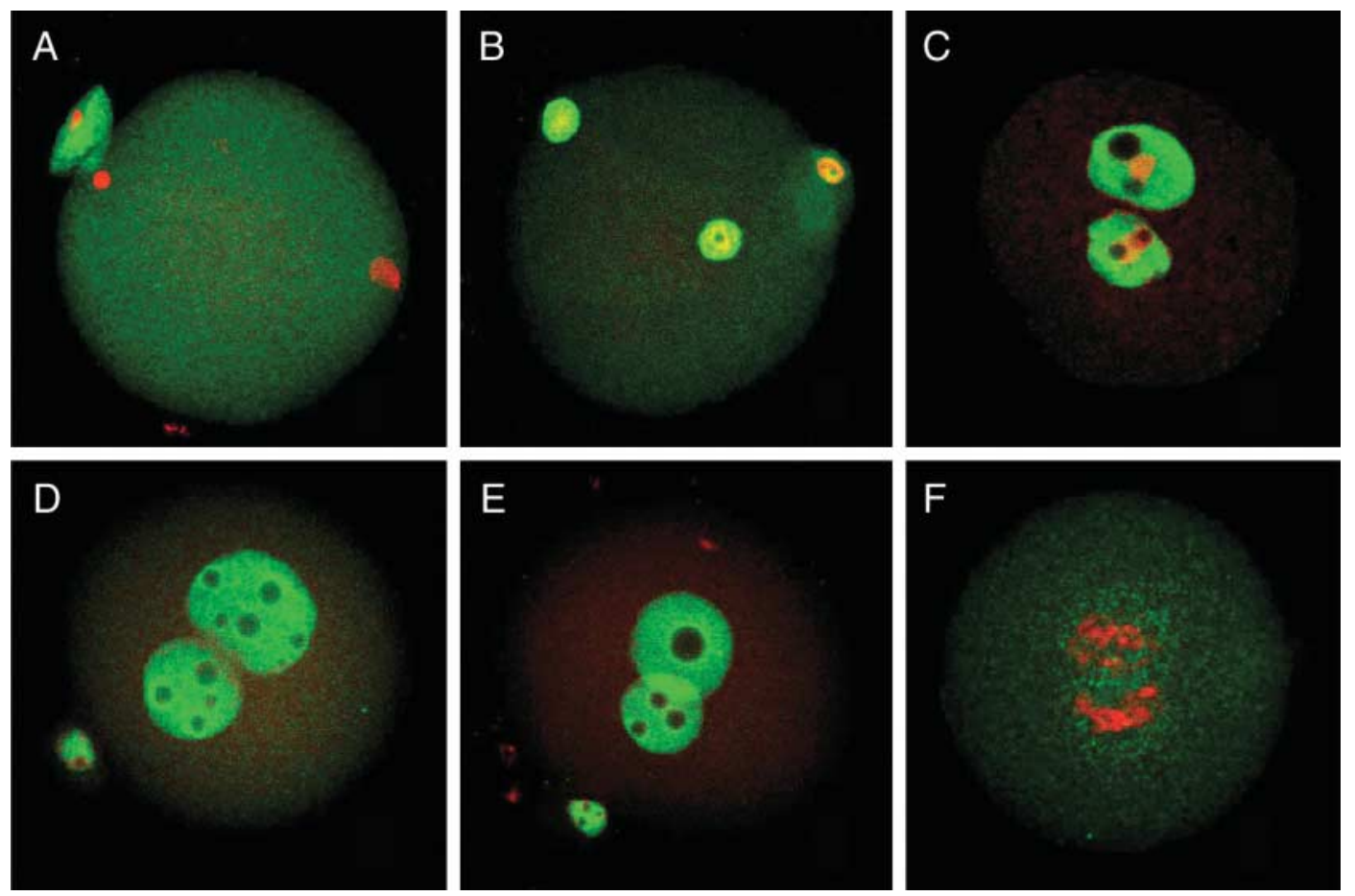

Figure 2 Localization of Ran after fertilization. Green, Ran; red, chromatin; yellow, overlapping of green and red. Ran was dispersed with the second polar body emission after sperm penetration (A) and concentrated in early male and female pronuclei (B). With the growth and apposition of the pronuclei the green fluorescent staining of Ran became stronger in the pronuclei than it was in the cytoplasm (C-E). After pronuclear membrane breakdown, Ran diffused through the entire zygote, with a stronger staining around condensed chromatin (F). Original magnification $\times 630$.

with the entire mitotic spindle (Fig. 3C). Then Ran concentrated between the separated chromosome clusters and formed two brighter green bands during anaphase and telophase of mitosis (Fig. 3D and E). After completion of the first mitotic cell cycle, two blastomeres formed and both of them entered interphase. At this time, Ran concentrated again in the assembling nuclei and the intensity of the nuclear staining of Ran compared with the cytoplasm became more evident during the completion of nuclear formation (Fig. $3 \mathrm{~F}$ and $\mathrm{G}$ ). Throughout the remainder of the cleavage stage, Ran showed the same pattern of dispersion: distribution to the cytoplasm at pro-metaphase, concentration to the spindle, localization between the separated chromosomes at telophase, and nuclear localization in the interphase nucleus (Fig. $3 \mathrm{H}-\mathrm{L}$ ).

\section{Localization of Ran when the microtubule organization was disturbed, the pseudo-cleavage was induced and the MII oocyte was activated with alcohol}

A total of $50 \mathrm{MIl}$ oocytes were treated with taxol, of which 25 were used for Ran detection, and the other 25 were used for $\alpha$-tubulin detection. We could find that in $100 \%$ of the oocytes Ran concentrated to the expanded spindle around the chromosomes (Fig. 4A), in the same configuration as microtubule distribution (Fig. 4B). Asters were observed in all taxol-treated oocytes, but we could not see any aster-like foci stained positively for Ran (Fig. 4A) as that shown for tubulin (Fig. 4B).

A total of 50 oocytes were treated with colchicine, a potent microtubule polymerization inhibitor, 25 were used for Ran detection and the other 25 were used for $\alpha$-tubulin detection. It was found that in $100 \%$ of the oocytes Ran protein distributed evenly in the cytoplasm, with a concentrated distribution beneath the cell membrane near the chromosomes (Fig. 4C), corresponding to the disappearance of the spindle (Fig. 4D).

When ZP-intact mouse oocytes at the GV stage were cultured overnight in the presence of both IBMX and $\mathrm{CB}, 82 \%$ (41 of 50 ) slowly underwent pseudo-cleavage, which included the division of the oocytes into two approximately equal portions, one of which contained an intact GV and nucleolus. Ran was localized mainly in the GV except for the nucleolus, and no staining was observed in the cytoplasm of the two cleaved portions (Fig. 4E).

When MII oocytes were activated by alcohol, and cultured for $6 \mathrm{~h}$ in M2 medium, Ran was concentrated to the pronuclei (Fig. 4F). 

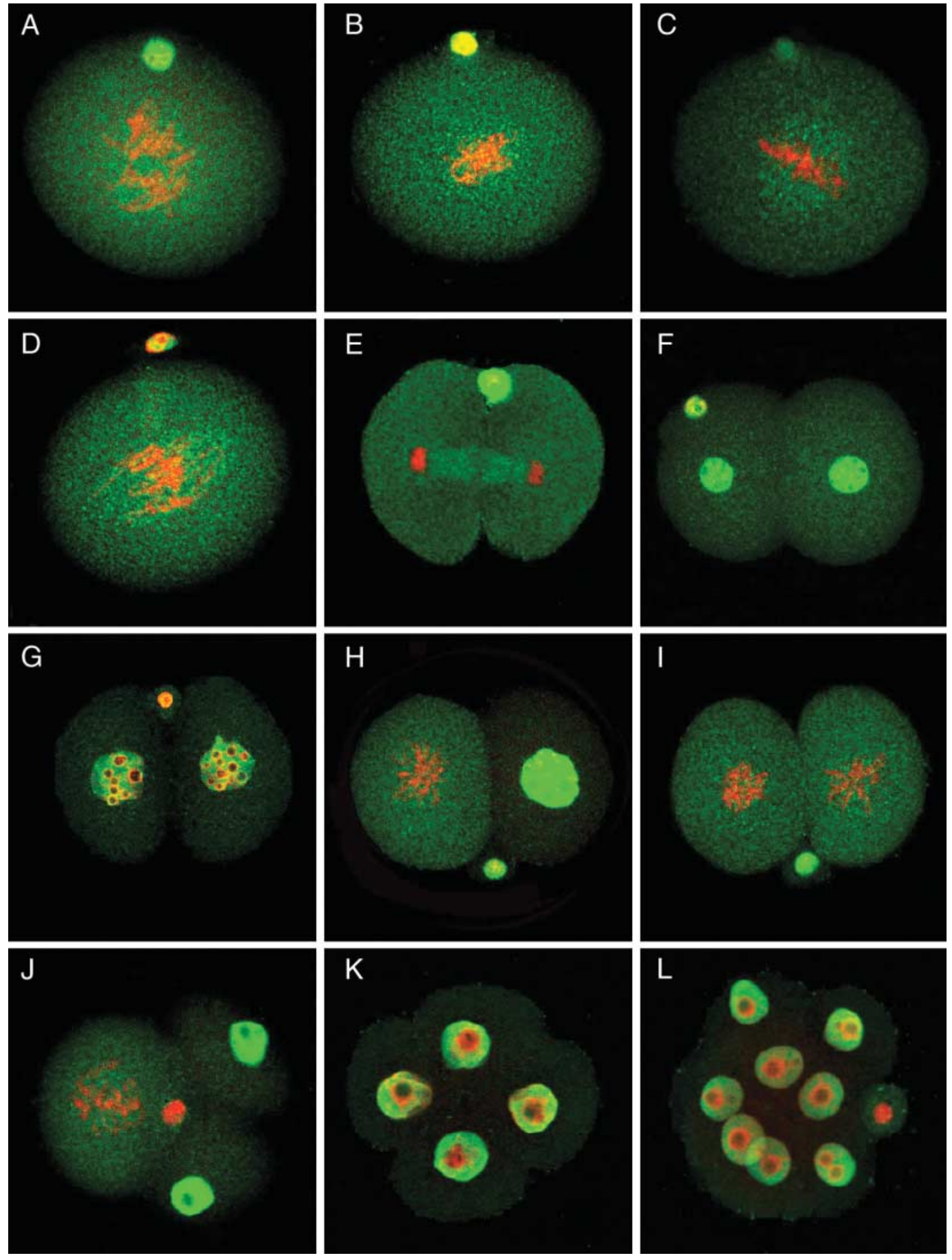

Figure 3 Localization of Ran during mitosis of early embryos. Green, Ran; red, chromatin; yellow, overlapping of green and red. Before first mitosis, Ran was preferentially stained in the converged chromosome region (A and B). With the organization of chromosomes to the equatorial plate, Ran staining associated with the entire mitotic spindle (C). Then Ran concentrated between the separated chromosomes during anaphase and telophase of mitosis ( $\mathrm{D}$ and $\mathrm{E}$ ). After completion of the first mitotic cell cycle, Ran concentrated again in the assembling nuclei ( $\mathrm{F}$ and $\mathrm{G}$ ). Following further cleavage, Ran showed the same pattern of dispersion: the distribution to the cytoplasm at pro-metaphase, the concentration to the spindle, localization between the separated chromosomes at telophase, and nuclear localization in the interphase nucleus $(\mathrm{H}-\mathrm{L})$. Original magnification $\times 630$. 

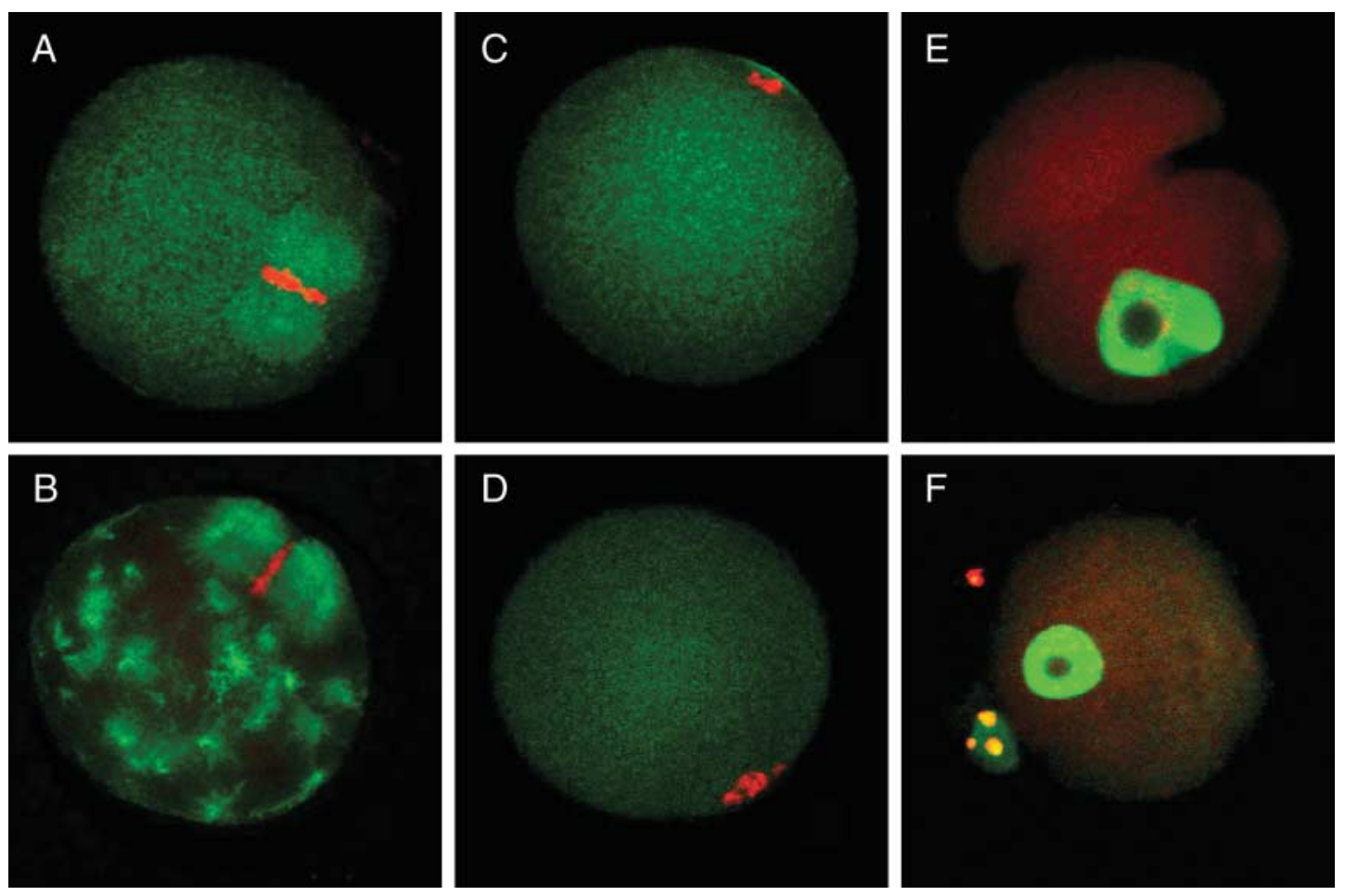

Figure 4 The distribution of Ran and $\alpha$-tubulin in mouse oocytes after different treatments. Green, Ran in (A), (C), (E) and (F) and tubulin in (B) and (D); red, chromatin; yellow, overlapping of green and red. After MII oocytes were treated with taxol; Ran concentrated to the expanded spindle around the chromosomes (A); many microtubule asters formed in the cytoplasm and the spindle expanded (B). When the eggs were treated with colchicine, Ran protein distributed evenly in the cytoplasm, with a concentrated distribution beneath the cell membrane near the chromosomes (C), corresponding to the disappearance of the spindle (D). In the pseudo-cleavage experiment, Ran was localized mainly in the GV except the nucleolus, and no staining was observed in the cytoplasm of the two cleaved portions (E). When MII oocytes were activated by alcohol, Ran was concentrated to the pronuclei $(\mathrm{F})$. Original magnification $\times 630$.

\section{Microinjection of anti-Ran antibody affects cell cycle progression and spindle assembly}

Interphase (GV-intact) oocytes were injected with anti-Ran antibody or with non-specific $\lg \mathrm{G}$ for control, then observed by light-field microscopy 2 and $14 \mathrm{~h}$ after injection; a proportion were fixed after $8 \mathrm{~h}$ and stained for confocal microscopy. As shown in Table 1, the GVBD rate of oocytes $2 \mathrm{~h}$ after antibody injection was significantly lower than that of the IgG-injected control group. The rate of the first polar body extrusion $14 \mathrm{~h}$ after antibody injection was significantly lower than that of the IgG-injected control group. Other oocytes did not extrude the first polar body, even when cultured for 16-18 h. After $8 \mathrm{~h}$ culture, abnormal spindles were observed in most oocytes injected. The spindles were significantly enlarged and the chromosomes could not be organized to the equatorial plate (Fig. 5A). A few oocytes did not even form spindles (Fig. 5B). At $14 \mathrm{~h}$, half of the oocytes (14 of 27) with the first polar body extruded showed abnormal spindles with broader poles (Fig. 5C) compared with controls (Fig. 5D).

\section{Discussion}

The Ran GTPase plays a central function in control of nucleo-cytoplasmic transport in interphase. In the present

Table 1 Effect on anti-Ran antibody microinjection on cell cycle progression and spindle assembly during mouse occyte maturation. Antibody was injected at the GV stage, GVBD and polar body emission were observed at 2 and $14 \mathrm{~h}$ respectively under light microscopy. Spindle assembly was observed by confocal microscopy $8 \mathrm{~h}$ after injection after immunostaining.

\begin{tabular}{lccc}
\hline & \multicolumn{2}{c}{ Cell cycle and spindle } \\
\cline { 2 - 4 } Group & $\begin{array}{c}\text { Percent (no.) of oocytes undergoing } \\
\text { GVBD at 2 h }\end{array}$ & $\begin{array}{c}\text { Percent (no.) of oocytes with } \\
\text { first polar body at 14 }\end{array}$ & $\begin{array}{c}\text { Percent (no.) of oocytes with abnormal } \\
\text { spindle or without spindle at } 8 \mathrm{~h}\end{array}$ \\
\hline Antibody microinjected & $65.2(58 / 89)^{\mathrm{a}}$ & $30.3(27 / 89)^{\mathrm{a}}$ & $91.2(31 / 34)^{\mathrm{a}}$ \\
Goat IgG microinjected & $81.0(85 / 105)^{\mathrm{b}}$ & $63.8(67 / 105)^{\mathrm{b}}$ & $34.3(12 / 35)^{\mathrm{b}}$ \\
\hline
\end{tabular}

In the same column, a vs b, $P<0.05$. 

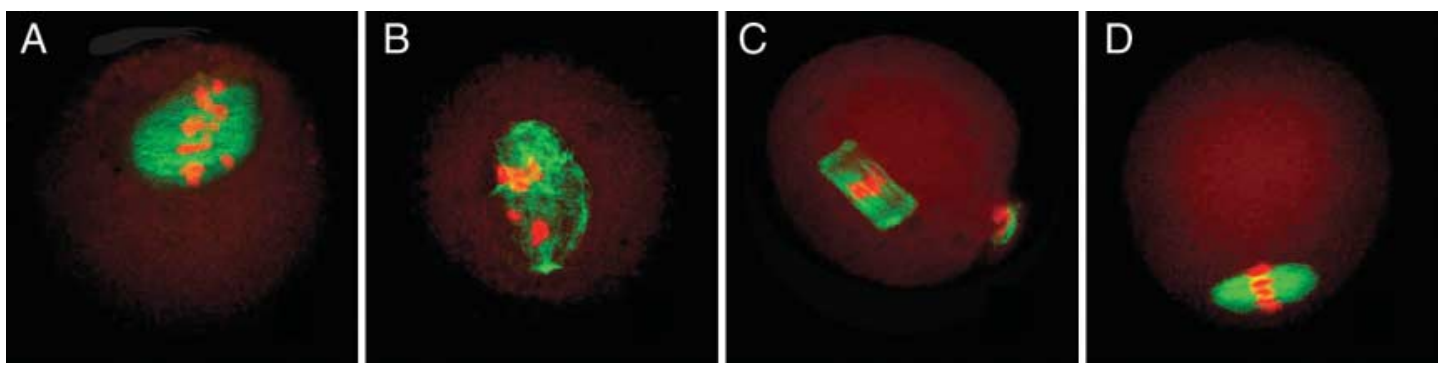

Figure 5 Effects of anti-Ran antibody injection on spindle organization. Green, tubulin; red, chromatin; yellow, overlapping of green and red. When the injected GV oocytes were cultured for $8 \mathrm{~h}$, abnormal spindles were observed in most injected oocytes, the spindles were much bigger and the chromosomes could not be organized to the equatorial plate (A). A few oocytes did not form spindles (B). The oocytes with the first polar body extruded showed abnormal spindles with broader poles $(C)$ when compared with the controls (D). Original magnification $\times 630$.

study, we found that Ran was localized mainly to the entire GV of mouse oocyte, to the male and female pronuclei after fertilization and to the nucleus of mouse early embryo cells in interphase, except for the nucleolus. Its concentration in the cytoplasm was too low to be observed. Ran was shown by immunofluorescence to localize to the nuclear envelope during interphase in early embryos of $C$. elegans (Bamba et al. 2002). It had been demonstrated that the Ran GTPase system, comprising RCC1, RanBP1, Ran and RanGAP, was essential for nucleo-cytoplasmic trafficking in C. elegans (Bamba et al. 2002). Ran drives nucleo-cytoplasmic transportation, depending upon the nature of its bound guanine nucleotide. Specificity of binding (i.e. to import or to export receptors) and the direction of movement are determined by where Ran is bound to GTP or GDP. The nucleo-cytoplasmic transport is driven by the asymmetric distribution of the GTP-bound form of Ran, i.e. large quantities are concentrated in the nucleus compared with small quantities in the cytoplasm across the nuclear pores (Macara 2002). Computer modeling has predicted about a 500-fold free RanGTP gradient across the nuclear pores in eukaryotic cells in vivo. Although we could not demonstrate which guanine nucleotide was bound by Ran in our experiment, we could clearly see the concentration of Ran in the nucleus. When anti-Ran antibody was microinjected into the cytoplasm of GV oocytes, GVBD was decreased. We suppose that the microinjected antibody affected the normal nucleo-cytoplasmic trafficking of some factors that affect the activation of maturation promoting factor, and thus partly inhibited GVBD.

During mouse oocyte maturation, the spatial organization of the microtubule cytoskeleton undergoes profound changes. The mitotic roles of Ran have been firmly established in Xenopus oocyte extracts. In our experiments, Ran was freed from the nucleoplasm to the cytoplasm and aggregated around the chromosomes. With the establishment of a bipolar spindle, it was obvious that the Ran distribution pattern was similar to that of the spindle microtubules. Ran was shown to be associated with the chromosomes in Xenopus, starfish and mouse oocytes (Hinkle et al. 2002). Our result is different from Ran's immunofluorescent staining in C. elegans where Ran localizes to kinetochore regions of metaphase and anaphase chromosomes (Bamba et al. 2002). This may be because Ran concentrated in the spindle in mouse oocytes, which could prevent us seeing its localization on the kinetochore region clearly. Experiments using Xenopus egg extracts showed that generation of RanGTP by RCC1 played roles in mitotic spindle assembly. In somatic cells, when Ran network members, such as RCC1 (Moore et al. 2002), RanBP1 (Di Fiore et al. 2003) or targets (TPX2) (Gruss et al. 2002) were experimentally perturbed, the spindle pole formation was primarily affected. After antiRan antibody was injected into the GV oocytes, we found abnormally large spindles without poles or with broad poles and the chromosomes could not be organized at the equatorial plate. In addition, some oocytes did not form spindles. The changes in spindle morphology are like those found in a TPX2 (a major regulatory target of Ran GTPase)-immunodepleted Xenopus system (Wittmann et al. 2000). Ran plays a central role in spatial and temporal organization microtubules of the vertebrate cell (Dasso 2002). Some Ran network members regulated by ubiquitin-like protein SUMO-1 (Joseph et al. 2002) or NPC subunit Nup358 (Salina et al. 2003, Joseph et al. 2004), localize transiently at kinetochores and act in the spindle attachment checkpoint. In our experiment, about one-third of injected oocytes eventually extruded polar bodies, suggesting that the spindle in these oocytes remains functional, while the others did not extrude a polar body in prolonged culture, which may be caused by the spindle's functional failure. The abnormal chromosome alignment and subsequent non-disjunction of chromosomes caused by Ran depletion may contribute the failure of cytokinesis.

Mouse oocytes and early embryos lack centrioles until the blastocyst stage. When microtubule organization was disturbed by taxol in mouse MII oocytes, all taxol-treated oocytes stained for $\alpha$-tubulin showed an expanded spindle and many aster-like foci in the cytoplasm, consistent with $\gamma$-tubulin distribution previously reported (Gueth-Hallonet et al. 1993). A fraction of Ran had been shown tightly associated with the centrosome throughout the cell cycle in HeLa cells, suggesting that Ran participated in the 
control of centrosome activity (Guy et al. 2003). The concentrated Ran distribution was only observed in the expanded spindle, while no clear distribution was observed in aster-like foci in our study. This may be because the antibody we used could not distinguish GTPand GDP-bound Ran and/or RanGTP concentration in aster-like foci was not high enough. When oocytes were treated with colchicine, the typical Ran distribution disappeared. The results provide further evidence showing the important roles of Ran in meiotic spindle microtubule assembly.

Ran has been implicated in cell cycle control, including the transition from mitosis (or meiosis) to interphase when the compartmentalization of the nucleus is established. In an experiment using a cell-free system of Xenopus egg extracts to examine the function of Ran during the assembly of pronuclei from demembranated Xenopus sperm heads, it was found that increased concentration of RanGTP produced a large, stable microtubule aster nucleated from the sperm centrosome and arrested nuclear assembly, blocking chromatin decondensation. In contrast to RanGTP, excess RanGDP had no inhibitory effects on aster formation and nuclear assembly. The RanGDP/GTP switch may play a role in co-ordinating changes in the structure of microtubules and the re-assembly of the nucleus associated with the transition from mitosis (or meiosis) to interphase (Zhang et al. 1999).

We found that Ran was concentrated in the pronuclei in fertilized mouse eggs and in the nuclei of early embryos. Ran's concentration in the interphase nuclei is either required directly to allow nuclear envelope assembly/disassembly to occur, or needed to modify chromatin. Ran and its cofactors RanBP2, RanGAP and RCC1 are all essential for reformation of the nuclear envelope after cell division. Reducing the expression of any of these components of the Ran GTPase cycle by RNAi leads to strong extranuclear clustering of integral nuclear envelope proteins and nucleoporins (Askjaer et al. 2002). Depleting Ran from $C$. elegans embryos by RNAi leads to defective nuclear envelope assembly (Kahana \& Cleveland 1999). Ran's direct role in nuclear envelope vesicle fusion came from experiments in which beads coupled to wild-type Ran could nucleate the assembly of functional, NPC-containing nuclear envelopes in extracts of either Xenopus eggs or mammalian cultured cells (Zhang \& Clark 2000). Recent data have shown that Ran associated with meiotic or mitotic chromatin both in nuclear assembly extracts in vitro and in several types of cells in vivo (Hetzer et al. 2002). In Xenopus egg extracts, it has been shown that RanGDP is essential for proper nuclear assembly and DNA replication (Hughes et al. 1998). The involvement of Ran in pronucleus formation from a very early stage as revealed in this study suggests that RanGDP may promote pronuclear formation in fertilized eggs. Further studies are required to prove this hypothesis.

The phenomenon of Ran's concentration in the nucleoplasm seems to require abundant nuclear binding sites, which apparently largely originate from the importin $\beta$ family transport receptor (Ribbeck et al. 1998, Zhang et al. 2002). In our experiments we observed Ran increased in intensity within the pronuclei with the growth and apposition of pronuclei, while Ran was almost absent in the cytoplasm. This also occurred in parthenogenetically activated eggs. When the chromosomes began to recondense just before pronuclear envelope breakdown, Ran accumulated in the region where the two pronuclei mixed. During first and second mitosis, the subcellular localization of concentrated Ran was associated with the chromatin and spindle microtubules throughout the entire cell cycle, just like its distribution pattern during meiosis. These results showed that Ran could regulate microtubule assembly during early mitosis of mouse embryo development. Ran did not show any specific distribution in meiotic and mitotic furrows as well as in pseudo-cleaved oocytes induced by IBMX and $\mathrm{CB}$ treatment, suggesting that it does not play functional roles in cytoplasmic division (cytokinesis).

Taken together, Ran has a cell cycle-dependent localization and may have regulatory roles in cell cycle progression and microtubule organization in mouse oocytes, fertilized eggs and early embryos.

\section{Acknowledgements}

This study was supported by the Grants from the National Natural Science Foundation of China (Nos 30225010, 30430530, 30170358) and Special Funds for Major State Basic Research ('973') Project of China (G1999055902). The authors declare that there is no conflict of interest that would prejudice the impartiality of this scientific work.

\section{References}

Askjaer P, Galy V, Hannak E \& Mattaj I 2002 Ran GTPase cycle and importins alpha and beta are essential for spindle formation and nuclear envelope assembly in living Caenorhabditis elegans embryos. Molecular Biology of the Cell 13 4355-4370.

Bamba C, Bobinnec Y, Fukuda M \& Nishida E 2002 The GTPase Ran regulates chromosome positioning and nuclear envelope assembly in vivo. Current Biology 12 503-507.

Bischoff F \& Ponstingl H 1991a Catalysis of guanine nucleotide exchange on Ran by the mitotic regulator RCC1. Nature 354 80-82.

Bischoff F \& Ponstingl H 1991b Mitotic regulator protein RCC1 is complexed with a nuclear Ras-related polypeptide. PNAS $\mathbf{8 8}$ 10830-10834.

Carazo-Salas R, Guarguaglini G, Gruss O, Segref A, Karsenti E \& Mattaj I 1999 Generation of GTP-bound Ran by RCC1 is required for chromatin-induced mitotic spindle formation. Nature $\mathbf{4 0 0}$ 178-181.

Compton D 2000 Spindle assembly in animal cells. Annual Review of Biochemistry 69 95-114.

Dai Y, Lee C, Hutchings A, Sun Y \& Moor R 2000 Selective requirement for $\mathrm{Cdc} 25 \mathrm{C}$ protein synthesis during meiotic progression in porcine oocytes. Biology of Reproduction 62 519-532.

Dasso M 2001 Running on Ran: nuclear transport and the mitotic spindle. Cell $104321-324$.

Dasso M 2002 The Ran GTPase: theme and variations. Current Biology 12 R502-R508. 
Di Fiore B, Ciciarello $M$, Mangiacasale $R$, Palena A, Tassin A, Cundari E \& Lavia P 2003 Mammalian RanBP1 regulates centrosome cohesion during mitosis. Journal of Cell Science $\mathbf{1 1 6}$ 3399-3411.

Drivas G, Shih A, Coutavas E, Rush M \& D'Eustachio P 1990 Characterization of four novel Ras-like genes expressed in a human teratocarcinoma cell line. Molecular Cell Biology 10 1793-1798.

Fleig U, Salus S, Karig I \& Sazer S 2000 The fission yeast Ran GTPase is required for microtubule integrity. Journal of Cell Biology 151 $1101-1111$.

Gorlich D \& Kutay U 1999 Transport between the cell nucleus and the cytoplasm. Annual Review of Cell and Developmental Biology $151101-1112$

Gruss O, Wittmann M, Yokoyama H, Pepperkok R, Kufer T, Sillje H, Karsenti E, Mattaj I \& Vernos I 2002 Chromosome-induced microtubule assembly mediated by TPX2 is required for spindle formation in HeLa cells. Nature Cell Biology 4 871-879.

Gueth-Hallonet C, Antony C, Aghion J, Santa-Maria A, LajoieMazenc I, Wright M \& Maro B 1993 gamma-Tubulin is present in acentriolar MTOCs during early mouse development. Journal of Cell Science 105 157-166.

Guy K, Barbara D, Claude C, Ferdinand L, Mette M, Annie D et al. 2003 Part of Ran Is Associated with AKAP450 at the centrosome: involvement in microtubule-organizing activity. Molecular Biology of the Cell $104260-4271$.

Hetzer M, Bilbao-Cortes D, Walther T, Gruss O \& Mattaj I 2000 GTP hydrolysis by Ran is required for nuclear envelope assembly. Molecular Cell 5 1013-1024.

Hetzer M, Gruss O \& Mattaj I 2002 The Ran GTPase as a marker of chromosome position in spindle formation and nuclear envelope assembly. Nature Cell Biology 4 E177-E184.

Hinkle B, Slepchenko B, Rolls M, Walther T, Stein P, Mehlmann L et al. 2002 Chromosomal association of Ran during meiotic and mitotic divisions. Journal of Cell Science 115 4685-4693.

Hughes M, Zhang C, Avis J, Hutchison C \& Clarke P 1998 The role of the ran GTPase in nuclear assembly and DNA replication: characterisation of the effects of Ran mutants. Journal of Cell Science 111 3017-3026.

Joseph J, Tan S, Karpova T, McNally J \& Dasso M 2002 SUMO-1 targets RanGAP1 to kinetochores and mitotic spindles. Journal of Cell Biology 156 595-602.

Joseph J, Liu S, Jablonski S, Yen T \& Dasso M 2004 The RanGAP1RanBP2 complex is essential for microtubule-kinetochore interactions in vivo. Current Biology 14 611-617.

Kahana J \& Cleveland D 1999 Beyond nuclear transport. Ran-GTP as a determinant of spindle assembly. Journal of Cell Biology 146 $1205-1210$.

Kalab P, Pu R \& Dasso M 1999 The Ran GTPase regulates mitotic spindle assembly. Current Biology 9 481-484.

Lounsbury K, Beddow A \& Macara I 1994 A family of proteins that stabilize the Ran/TC4 GTPase in its GTP-bound conformation. Journal of Biological Chemistry 269 11285-11290.

Macara I 2002 Why FRET about Ran? Developmental Cell 2 379-380.

Matsumoto T \& Beach D 1991 Premature initiation of mitosis in yeast lacking RCC1 or an interacting GTPase. Cell 66 347-360.
Melchior F, Guan T, Yokoyama N, Nishimoto T \& Gerace L 1995 GTP hydrolysis by Ran occurs at the nuclear pore complex in an early step of protein import. Journal of Cell Biology 131 $571-581$.

Moore W, Zhang C \& Clarke P 2002 Targeting of RCC1 to chromosomes is required for proper mitotic spindle assembly in human cells. Current Biology 12 1442-1447.

Ohba T, Nakamura M, Nishitani H \& Nishimoto T 1999 Self-organization of microtubule asters induced in Xenopus egg extracts by GTP-bound Ran. Science 284 1356-1358.

Ouspenski I 1998 A RanBP1 mutation which does not visibly affect nuclear import may reveal additional functions of the Ran GTPase system. Experimental Cell Research 244 171-183.

Quimby B, Wilson C \& Corbett A 2000 The interaction between Ran and NTF2 is required for cell cycle progression. Molecular Biology of the Cell 11 2617-2629.

Ren M, Coutavas E, D'Eustachio P \& Rush M 1994 Effects of mutant Ran/TC4 proteins on cell cycle progression. Molecular and Cellular Biology 14 4216-4224.

Ribbeck K, Lipowsky G, Kent H, Stewart M \& Gorlich D 1998 NTF2 mediates nuclear import of Ran. EMBO Journal 17 6587-6598.

Salina D, Enarson P, Rattner J \& Burke B 2003 Nup358 integrates nuclear envelope breakdown with kinetochore assembly. Journal of Cell Biology 162 991-1001.

Sazer S \& Dasso M 2000 The Ran decathlon: multiple roles of Ran. Journal of Cell Science 113 1111-1118.

Takai Y, Sasaki T \& Matozaki T 2001 Small GTP-binding proteins. Physiological Reviews 81 153-208.

Wassarman PM, Ukena TE, Josefowicz WJ, Letourneau GE \& Karnovsky MJ 1977 Cytochalasin B-induced pseudo-cleavage of mouse oocytes in vitro. II. Studies of the mechanism and morphological consequences of pseudocleavage. Journal of Cell Science $26323-337$.

Wittmann T, Wilm M, Karsenti E \& Vernos I 2000 TPX2, a novel Xenopus MAP involved in spindle pole organization. Journal of Cell Biology 149 1405-1418.

Wittmann T, Hyman A \& Desai A 2001 The spindle: a dynamic assembly of microtubules and motors. Nature Cell Biology $\mathbf{3}$ $28-34$.

Zhang C \& Clark P 2000 Chromatin-independent nuclear envelope assembly induced by Ran GTPase in Xenopus egg extracts. Science 288 1429-1432.

Zhang C, Hughes M \& Clarke P 1999 Ran-GTP stabilises microtubule asters and inhibits nuclear assembly in Xenopus egg extracts. Journal of Cell Science 112 2453-2461.

Zhang C, Hutchins J, Muhlhausser P, Kutay U \& Clarke P 2002 Role of importin-beta in the control of nuclear envelope assembly by Ran. Current Biology 12 498-502.

Received 22 February 2005

First decision 14 March 2005

Accepted 18 March 2005 